\title{
Radiometric Characterization and Absolute Calibration of the Marine Optical System (MOS) Bench Unit
}

\author{
Catherine Habauzit, Steven W. Brown, Keith R. Lykke, and B. Carol Johnson \\ Optical Technology Division, National Institute of Standards and Technology, Gaithersburg, Maryland \\ Michael E. Feinholz and Mark Yarbrough \\ Moss Landing Marine Laboratories, Moss Landing, California \\ DenNis K. Clark \\ National Oceanic and Atmospheric Administration/National Environmental Satellite, Data, and Information Service, \\ Camp Springs, Maryland
}

(Manuscript received 15 January 2002, in final form 9 October 2002)

\begin{abstract}
The Marine Optical System (MOS) is a dual charge-coupled device (CCD)-based spectrograph system developed for in-water measurements of downwelling solar irradiance $E_{d}$ and upwelling radiance $L_{u}$. These measurements are currently used in the calibration and validation of satellite ocean color measurement instruments such as the moderate resolution imaging spectroradiometer (MODIS) and the Sea-viewing Wide Field-of-view Sensor (SeaWiFS). MOS was designed to be deployed from a ship for single measurements and also integrated into the Marine Optical Buoy (MOBY) for longer time series datasets. Measurements with the two spectrographs in the MOS systems can be compared in the spectral interval from about 580 to $630 \mathrm{~nm}$. In this spectral range, they give different values for $L_{u}$ or $E_{d}$ at a common wavelength. To better understand the origin of this observation and the sources of uncertainty in the calibration of MOBY, an MOS bench unit was developed for detailed radiometric characterization and calibration measurements in a laboratory setting. In the work reported here, a novel calibration approach is described that uses a tunable-laser-based, monochromatic, spatially uniform, Lambertian, large area integrating sphere source (ISS). Results are compared with those obtained by a conventional approach using a lamp-illuminated ISS. Differences in the MOS bench unit responsivity between the two calibration approaches were observed and attributed to stray light. A simple correction algorithm was developed for the lamp-illuminated ISS that greatly improves the agreement between the two techniques. Implications for water-leaving radiance measurements by MOS are discussed.
\end{abstract}

\section{Introduction}

The National Aeronautics and Space Administration (NASA) and other government agencies have developed a series of remote sensing programs that study properties of the earth on a global scale. Information about the atmosphere, oceans, or land, such as species concentration (ozone, chlorophyll, etc.) or temperatures, is derived from measurements of the reflected or emitted energy from the earth by satellite-based instruments (Elachi 1987). Ground-based sensors are often used to determine detailed information about the radiometric properties of the surface and atmosphere, which is then compared with measurements by the satellite sensors.

Corresponding author address: Dr. B. Carol Johnson, U.S. Department of Commerce, National Institute of Standards and Technology, 100 Bureau Dr., MS 8441, Gaithersburg, MD 20899-8441. E-mail: cjohnson@nist.gov
In general, if the ground-based results are sufficiently accurate, the information can be used to provide a revised calibration of the satellite sensor. By repeating the measurements over the lifetime of the satellite sensor, its responsivity can be recorded as a function of time.

However, for ocean color remote sensing, it is always necessary to calibrate the satellite sensor using in situ results, independent of the status of the preflight radiometric calibration or the utility of onboard calibration strategies (Gordon 1998). This is because, for ocean waters, the atmosphere is responsible for the majority of the at-satellite radiance, and its characterization is a critical part of the analysis. The Marine Optical Buoy (MOBY; Clark et al. 2002a,b, 1997) has been deployed in support of the Earth Observing System (EOS) since 1996 and is used as a calibration reference station for ocean color satellite instruments. The marine environment (off the coast of Lanai, Hawaii) was selected to 
simplify the application of the atmospheric correction algorithms (Clark et al. 1997).

MOBY uses a charge-coupled device (CCD)-based dual spectrograph known as the Marine Optical System (MOS) as a sensor connected to fiber optics to measure the upwelling radiance $L_{u}$ (scattered by the ocean), as well as the downwelling irradiance $E_{d}$ (coming from the sun), at different depths in the ocean. As described in Clark et al. (2002b), these data are used to determine the water-leaving radiance $L_{w}$. The MOBY measurements are made with sufficient wavelength coverage and spectral resolution to be used by a wide variety of ocean color satellite sensors. For these sensors, the spectral parameters are determined by interference filters with typical center wavelengths of 412, 443, 490, 555, and $670 \mathrm{~nm}$, and bandpasses from 10 to $20 \mathrm{~nm}$. Radiance ratios of these bands are used to estimate global concentrations of chlorophyll- $a$ and other pigments using relationships (bio-optical algorithms), which in turn are developed in separate studies (Gordon et al. 1980).

The vicarious calibration approach of the Sea-viewing Wide Field-of-view Sensor (SeaWiFS; Barnes and Holmes 1993) using MOBY data is described in detail in Barnes et al. (2000). For the moderate resolution imaging spectroradiometer (MODIS; Esaias et al. 1998), a similar procedure is followed (see Web site: http:// modis-ocean.gsfc.nasa.gov/). MOBY data are also used to support the comparison and merger of ocean color data from multiple sensors. For example, vicarious calibrations using MOBY for the Japanese Ocean Color Temperature Scanner (OCTS; Isaacman et al. 1999), and the French Polarization and Directionality of the Earth's Reflectances (POLDER; Deschamps et al. 1994) allowed for a thorough comparison of their ocean color data (Wang et al. 2002).

MOBY and other MOS systems (used during short cruises) are calibrated before and after deployment using sources traceable to radiometric standards maintained at the National Institute of Standards and Technology (NIST; Clark et al. 2002a). The secondary standards used for radiance are Integrating Sphere Sources (ISSs) that are externally illuminated by quartz halogen lamps. For irradiance, the sources are FEL-type, 1000-W quartz halogen lamps. Both types of sources are recalibrated based on lamp operating hours: the sphere sources by a commercial standards laboratory and the FEL lamps by NIST. The MOBY radiance and irradiance standards are monitored during the year using NIST-developed Standard Lamp Monitor (SLM) radiometers that are, in turn, calibrated at NIST on a regular basis. In addition, a portable transfer radiometer, the Visible Transfer Radiometer (VXR; Butler et al. 1999) and a calibrated EOS/NIST Portable Radiance (NPR) source (Brown and Johnson 2002) are deployed from NIST to the MOBY field laboratory on a regular basis to validate the calibration protocols and assess the accuracy and stability of the MOBY spectral radiance sources. The VXR and the NPR are calibrated at NIST. The resulting uncer- tainties for the radiometric calibration of in-water measurements, based on this calibration approach and without accounting for the effects of stray light, are estimated to be $4 \%$ to $8 \%(k=1)$ (Clark et al. 2002a).

The generally stated uncertainty goal for ocean color radiometry is $5 \%(k=1)$ for $L_{w}$, which results in an uncertainty of $35 \%$ for chlorophyll- $a$ (Hooker et al. 1993). This requirement for high accuracy, and the advent of more advanced remote sensing instrumentation, places stringent demands on the uncertainties in waterleaving radiance measurements with MOBY. Measurements with the two spectrographs in the MOS systems can be compared in the spectral interval from about 580 to $630 \mathrm{~nm}$. In this spectral range they give different values for $L_{u}$ or $E_{d}$ at a common wavelength. To better understand the origin of this observation and to better quantify the uncertainties in the calibration of MOBY, an MOS bench unit was developed for detailed radiometric characterization and calibration measurements in a laboratory setting.

In the work reported here, spectral radiance responsivity calibrations of one of the two spectrographs in the MOS bench unit using a lamp-based ISS are compared and contrasted with results using a monochromatic (and broadly tunable), uniform, Lambertian, and large area ISS. Differences in the MOS bench unit responsivity between the two approaches are attributed to stray light. The effect of stray light in ocean color research can be significant because the relative spectral shape of the lamp-based calibration source is quite different from the in-water case (Clark et al. 2002a).

\section{Optical configuration of the MOS bench unit}

A schematic diagram of the MOS bench unit is shown in Fig. 1a. For the radiance port, light is collected by a 3-cm-diameter lens and relayed to the dichroic beamsplitter using a plane mirror. The mirror is mounted to a shaft so that it rotates about an axis perpendicular to the plane of the paper. There are four mirror positions corresponding to coupling light from the radiance port, the irradiance port, an internal set of sources, and an occulted (dark) position. A dichroic beam splitter is mounted inside the column at a $45^{\circ}$ angle. At this incident angle, it transmits radiant flux with high efficiency from 700 to beyond $1000 \mathrm{~nm}$ while reflecting radiant flux in the spectral range from 400 to $650 \mathrm{~nm}$. The reflected flux is sent to one spectrograph, here called the blue spectrograph (BSG), and the transmitted flux to a second spectrograph called the red spectrograph (RSG). In the RSG, a 500-nm cut-on filter is used to eliminate any potential contribution from second-order diffraction of flux in the 350-400-nm spectral region that is transmitted through the dichroic beam splitter. For both spectrographs, a cylindrical lens focuses light onto a $25-\mu \mathrm{m}$-wide entrance slit (Fig. 1b). The light is then dispersed using a fixed convex holographic grating and imaged onto the CCD array. Dark current in the 


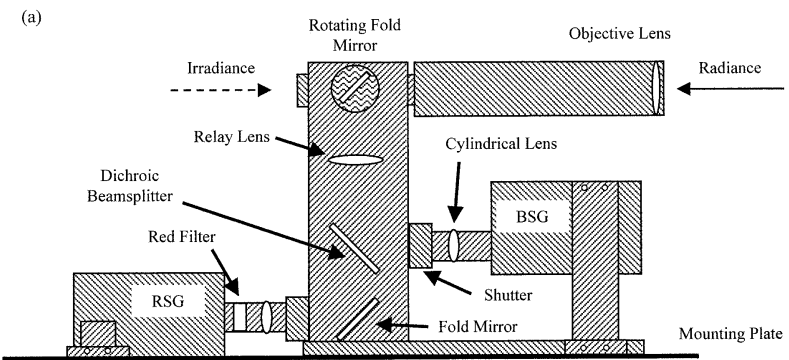

(b)

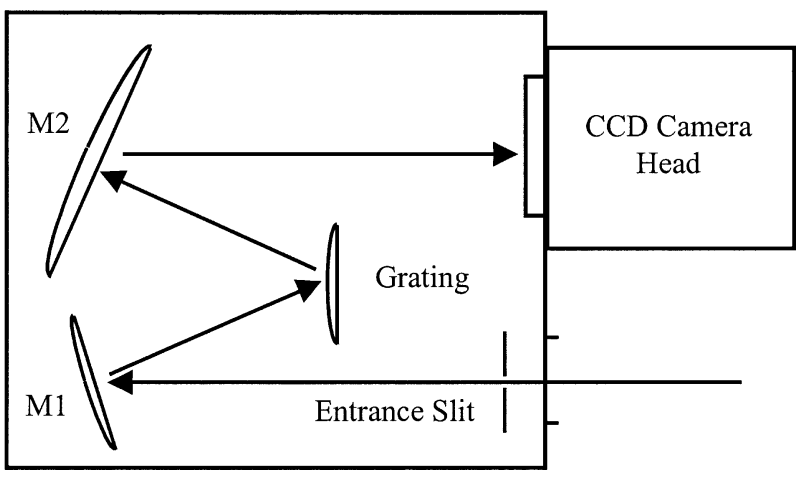

FIG. 1. (a) Simplified view of MOS bench unit configured for radiance measurements. The irradiance optics are not attached. The red spectrograph is labeled RSG and the blue spectrograph is labeled BSG. (b) Top view of one of the spectrographs, illustrating schematically the entrance slit, collimating mirror M1, fixed convex holographic grating, focusing mirror M2, and the CCD array detector. The physical dimensions are $14 \mathrm{~cm} \times 10 \mathrm{~cm} \times 6 \mathrm{~cm}$.

CCDs is reduced by cooling them to $-37^{\circ} \mathrm{C}$. The unsigned 16-bit images are acquired using commercial software, ${ }^{1}$ which also controls the exposure time. The number of analog-to-digital units (ADUs) is proportional to the number of electrons confined in each pixel.

\section{Calibration using tunable laser sources}

\section{a. Description of the SIRCUS facility}

A schematic diagram of the facility for spectral irradiance and radiance responsivity calibrations using uniform sources (SIRCUS) is shown in Fig. 2 (Brown et al. 2000). The output of a laser is directed into an intensity stabilizer that reduces the magnitude of incident power fluctuations to less than $0.1 \%$. A portion of the laser flux is directed into a wavemeter to measure the wavelength of the radiation with a standard uncertainty of $0.001 \mathrm{~nm}$. The remainder of the laser beam is

\footnotetext{
${ }^{1}$ The product used was IP Lab series 200 software, from Photometrics Ltd., 3440 East Britannia Drive, Tucson, Arizona 85706. Certain commercial equipment, instruments, or materials are identified in this paper to foster understanding. Such identification does not imply recommendation or endorsement by the National Institute of Standards and Technology, nor does it imply that the materials or equipment identified are necessarily the best available for the purpose.
}

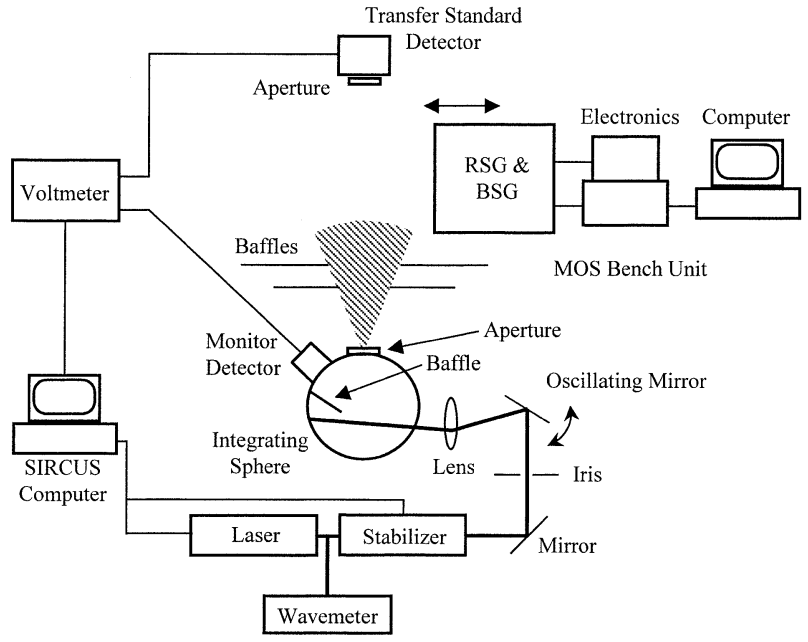

FIG. 2. Experimental configuration of the SIRCUS facility showing the general laser components, the laser-illuminated ISS, the monitor detector, the MOS bench unit, and the transfer standard detector.

directed into the integrating sphere after reflection from a vibrating mirror and focused by a $100-\mathrm{mm}$ focal lens onto a side entrance port in the sphere wall. The vibrating mirror serves to reduce the effect of speckle that occurs because of coherence properties of laser radiation, while the lens improves the uniformity of the radiance at the sphere exit port. A variety of fixed frequency and tunable lasers are used to cover the spectral range from 370 to $900 \mathrm{~nm}$. A Ti:sapphire laser provides tunable coverage from 690 to $900 \mathrm{~nm}$, and the frequency-doubled Ti:sapphire gives tunable coverage from 370 to $450 \mathrm{~nm}$. The spectral range from 450 to $550 \mathrm{~nm}$ is covered using fixed frequency lasers while dye lasers are used to cover the spectral region from 550 to $700 \mathrm{~nm}$.

There is a silicon monitor detector mounted on the wall of the ISS. Prior to calibrating the MOS bench unit, the monitor detector was calibrated against a reference standard detector so that the monitor detector signal is related to the ISS radiance over the wavelength range of interest. Neither detector is temperature-stabilized. Once calibrated, the sphere radiance is known at any wavelength by measuring the monitor detector signal. The MOS bench unit was mounted on a movable carriage and could be positioned in front of the exit sphere and moved out of the way when necessary (e.g., during the monitor calibration).

\section{b. MOS image}

This work focused on results from the RSG. In the MOS bench unit, light is dispersed along the CCD column direction (increasing wavelength corresponds to increasing column number). Consequently, we associate the individual columns in the $512 \times 512 \mathrm{CCD}$ array with the measurement wavelengths of the system. In the other dimension of the CCD array, the image of the slit 
(a)

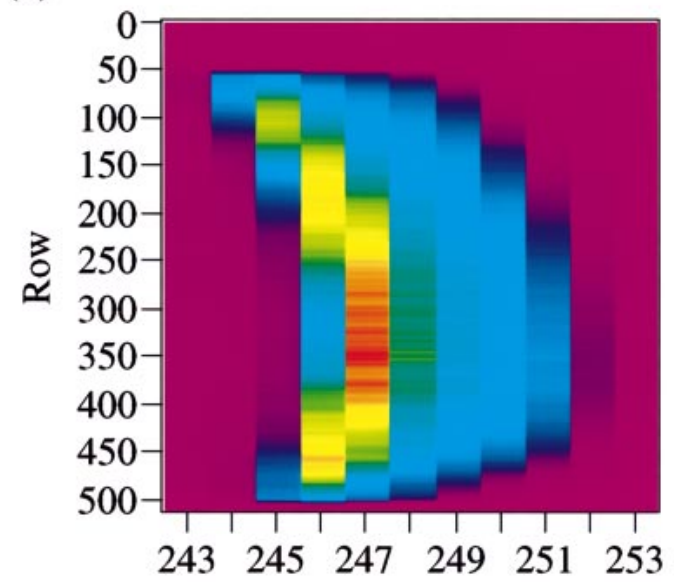

\section{Column}

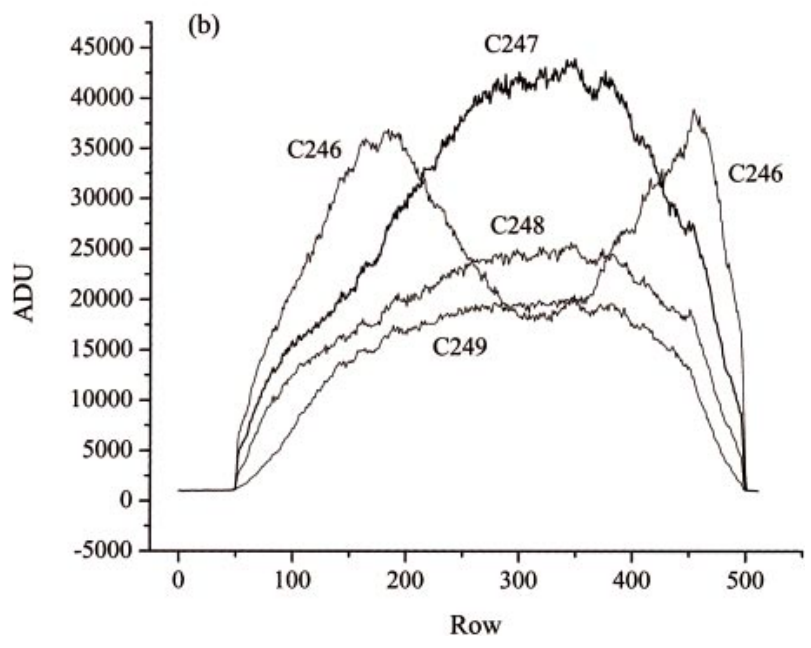

FIG. 3. (a) An image, using a rainbow color table, of the total counts for a 1-s exposure for the RSG illuminated with flux at 745 $\mathrm{nm}$ from SIRCUS. The maximum (red) was 44012 ADU, while the minimum (magenta), was 950 ADU and corresponded to the dark counts. (b) The variation in ADU with row number for four adjacent columns for the data in (a). The column identification is indicated.

is averaged over the rows. The image of the slit at a particular wavelength is spread out over several columns, as shown in Fig. 3a for incident flux at $745 \mathrm{~nm}$, and along most of the rows in the CCD. The image shows a nonuniform distribution within a column of pixels as well as a curvature of the slit image. In addition, in this bench unit, the image is not centered on the CCD. The effect of the curvature is illustrated in Fig. 3b, where the output as a function of a row pixel is shown for four adjacent columns. For a particular column, the variations observed with a row pixel could be attributed to scattering from dust particles at the spectrograph entrance slit. The curvature is most likely due
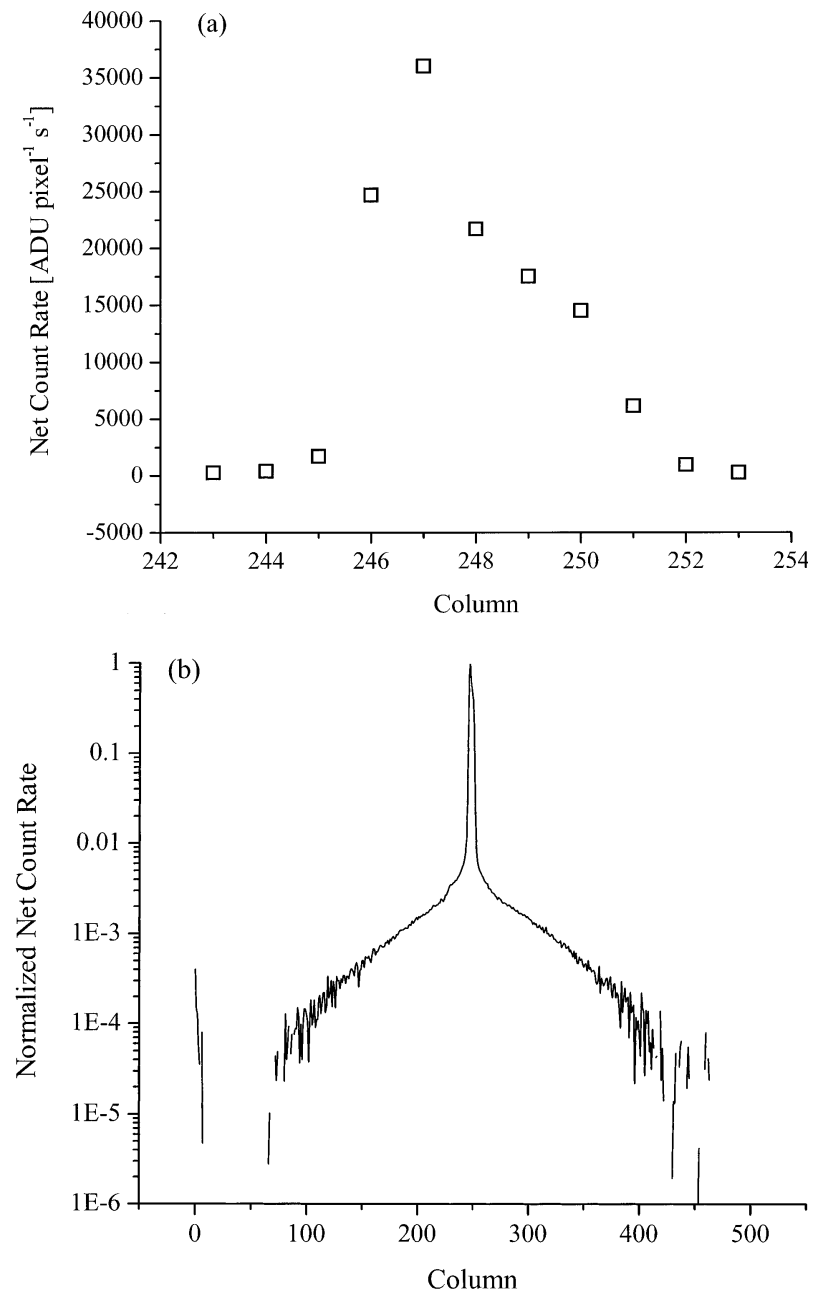

FIG. 4. (a) The net count rate averaged for rows 200-299, and shown for columns 243-253 for illumination of the RSG at $745 \mathrm{~nm}$. The data are the background subtracted, row average of those presented in Fig. 3. (b) Normalized net count rate, averaged for rows 200-299, for illumination at $745 \mathrm{~nm}$ for all of the columns in the CCD array. Negative values are omitted from the plot.

to optical aberrations and the CCD not being exactly at the focal plane of the spectrograph. As shown in Fig. $4 \mathrm{a}$, for $745-\mathrm{nm}$ excitation, the resultant net signal is concentrated mainly between columns 244 and 252 . However, as shown in Fig. 4b, there is a small, nonzero response across the entire array. This nonzero response from columns far from the column that has the maximum signal is due to stray light scattered by the optical components in the system. As will be discussed in detail later, consideration of stray light has important consequences for the calibration of MOS using broadband incandescent sources.

\section{c. Spectral responsivity}

The measurement equation relating the signal $S_{i}\left(\lambda_{i}\right)$ for column $i$ to the spectral radiance of the source $L_{\lambda}(\lambda)$ is given by (Kostkowski 1979) 


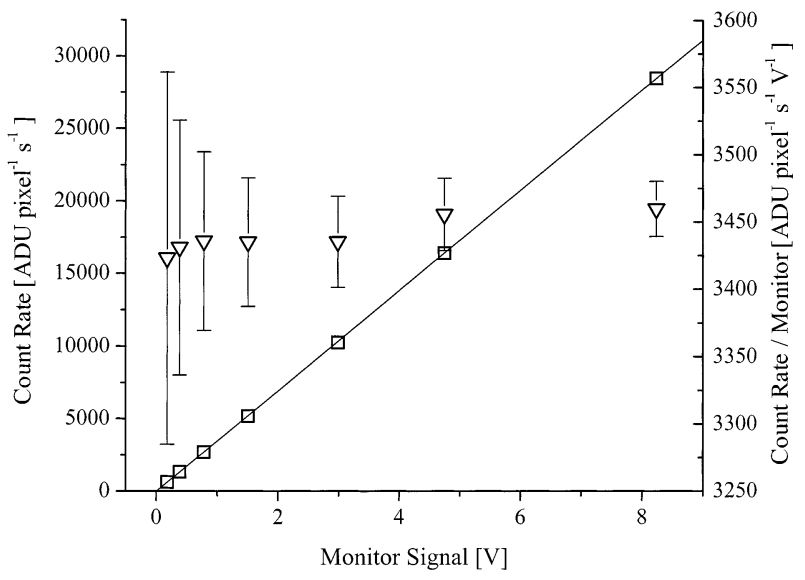

FIG. 5. The linearity of the RSG at $612 \mathrm{~nm}$ is shown as the average count rate as a function of monitor signal (squares), and normalized by the monitor signal (triangles). The line is a linear fit to the data.

$$
S_{i}\left(\lambda_{i}\right)=\int L_{\lambda}(\lambda) R_{i}\left(\lambda_{i}, \lambda\right) d \lambda .
$$

In Eq. (1), $\lambda_{i}$ is the wavelength associated with the peak response from column $i$; it is similar to the wavelength setting in a scanning monochromator. The column radiance responsivity function $R_{i}\left(\lambda_{i}, \lambda\right)$ describes the average response (over the slit height) of column $i$ to light at wavelength $\lambda$. For each column, $R_{i}\left(\lambda_{i}, \lambda\right)$ accounts for the system throughput at that location along the CCD array, the spectral response of the pixels in that column, and the stray light at wavelength $\lambda$ that is scattered or diffracted onto column $i$. For the MOS bench unit, the units of $R_{i}\left(\lambda_{i}, \lambda\right)$ are in ADU pixel ${ }^{-1} \mathrm{~s}^{-1} \mathrm{~W}^{-1} \mathrm{~cm}^{2} \mathrm{sr}$.

Because SIRCUS uses extremely narrowband laser sources, the column radiance responsivity function is constant over the integral indicated in Eq. (1); that is, $L_{\lambda}(\lambda) \cong L\left(\lambda^{\prime}\right) \delta\left(\lambda-\lambda^{\prime}\right)$. For a particular laser excitation wavelength $\lambda^{\prime}$,

$$
S_{i}\left(\lambda_{i}, \lambda^{\prime}\right)=L\left(\lambda^{\prime}\right) R_{i}\left(\lambda_{i}, \lambda^{\prime}\right),
$$

where $L\left(\lambda^{\prime}\right)$ is the radiance of the sphere source (in W $\mathrm{cm}^{-2} \mathrm{sr}^{-1}$ ) and $S\left(\lambda_{i}, \lambda^{\prime}\right)$ is the average count rate (in ADU pixel ${ }^{-1} \mathrm{~s}^{-1}$ ) for column $i$. The absolute radiance responsivities of any column in the array for a monochromatic source at $\lambda^{\prime}$ are determined from the expression

$$
R_{i}\left(\lambda_{i}, \lambda^{\prime}\right)=\frac{S_{i}\left(\lambda_{i}, \lambda^{\prime}\right)}{L\left(\lambda^{\prime}\right)} .
$$

By tuning the excitation wavelength, the absolute radiance responsivity of each column can be determined.

\section{d. SIRCUS results}

Before calculating the radiance responsivity, it was necessary to determine the linearity of the response and its dependence on exposure time. To evaluate the lin-

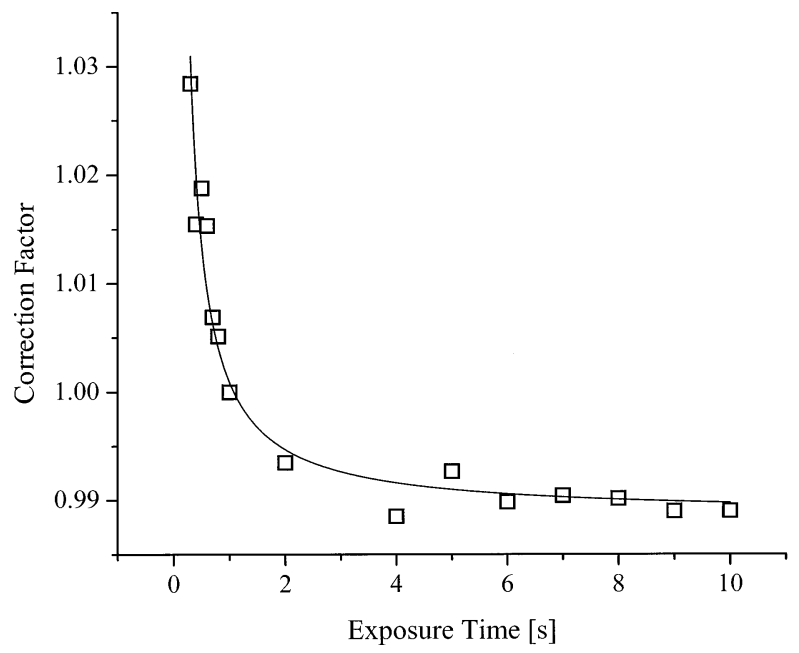

FIG. 6. The exposure time correction results for the RSG at $750 \mathrm{~nm}$ (squares) and the model fit to the data [solid line; see Eq. (5)].

earity of the system response, we measured the average count rate at $612 \mathrm{~nm}$ for a 1-s exposure time as the sphere radiance was changed. The area on the CCD array that was averaged included rows 150-350 and five columns around the column that had the maximum count rate. The results are shown in Fig. 5. The left ordinate is the average count rate as a function of monitor signal (which is directly proportional to the sphere radiance); the right ordinate is the average count rate divided by the monitor signal, giving a normalized response.

The uncertainties in Fig. 5 were calculated using the expression

$$
\Delta\left(\frac{S_{i}}{S_{\mathrm{mon}}}\right)=\frac{S_{i}}{S_{\mathrm{mon}}}\left[\left(\frac{\Delta S_{i}}{S_{i}}\right)^{2}+\left(\frac{\Delta S_{\mathrm{mon}}}{S_{\mathrm{mon}}}\right)^{2}\right]^{1 / 2},
$$

where $\Delta S_{i}$ is given by Poisson's distribution, $\Delta S_{i}=$ $\sqrt{S_{i}}$, and $\Delta S_{\text {mon }}$ is the standard deviation of the mean in the measurement of the monitor signal $S_{\text {mon }}$. The results show that the system response is linear to within the measurement uncertainties (which were between $2.8 \%$ and $0.6 \%$ ) over the entire radiance range measured.

We also measured, at $750 \mathrm{~nm}$, the CCD response as the exposure time was varied from 0.2 to $10 \mathrm{~s}$. The number of counts at column 253 was averaged over 100 rows (from 200 to 300). The radiance was changed along with the exposure time to keep the number of counts approximately constant in order to eliminate any possible nonlinear effects. The averaged number of counts, normalized to the monitor signal, was divided by the exposure time. This result was then normalized by the count rate at $1 \mathrm{~s}$ to get a value $y(t)$ for each exposure time $t$. The results, shown in Fig. 6, were modeled assuming there is a finite delay associated with the opening of the shutter and the delay is independent of exposure time. In terms of the normalized results shown in Fig. 6 , this model is 

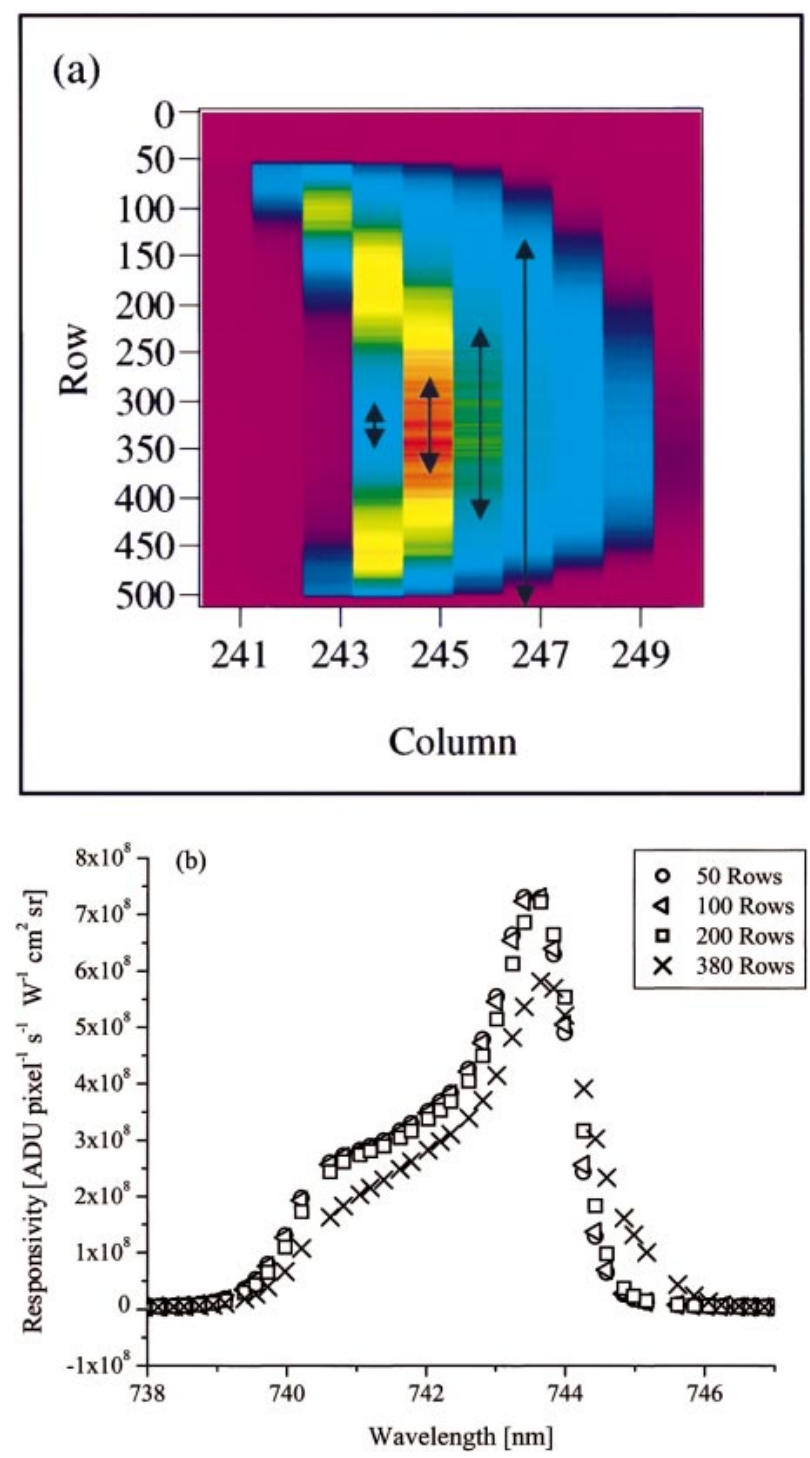

FIG. 7. (a) False color image of the total counts for the RSG illuminated at $743.4 \mathrm{~nm}$ for columns $241-250$ with vertical lines to indicate the areas used to determine the responsivity of column 245. (b) The responsivity of column 245 as a function of wavelength for averaging over the image of the slit using 50,100, 200, and 380 rows centered on row 325 .

$$
y=\frac{y_{0} t}{t-\Delta t}
$$

The delay $\Delta t$ and the constant $y_{0}$ were determined using a nonlinear least squares fitting procedure. The square of the correlation coefficient for the fit was 0.956, and the values and their standard uncertainties are $y_{0}=$ $0.9885 \pm 0.0010$ and $\Delta t=0.01235 \mathrm{~s} \pm 0.00072 \mathrm{~s}$. For the analysis in this work, the count rates were corrected to a 1-s exposure time.

It is clear from Fig. 3a that the image quality will affect the shape of $R_{i}\left(\lambda_{i}, \lambda^{\prime}\right)$ because of the asymmetry in the dispersion direction and the curvature along the

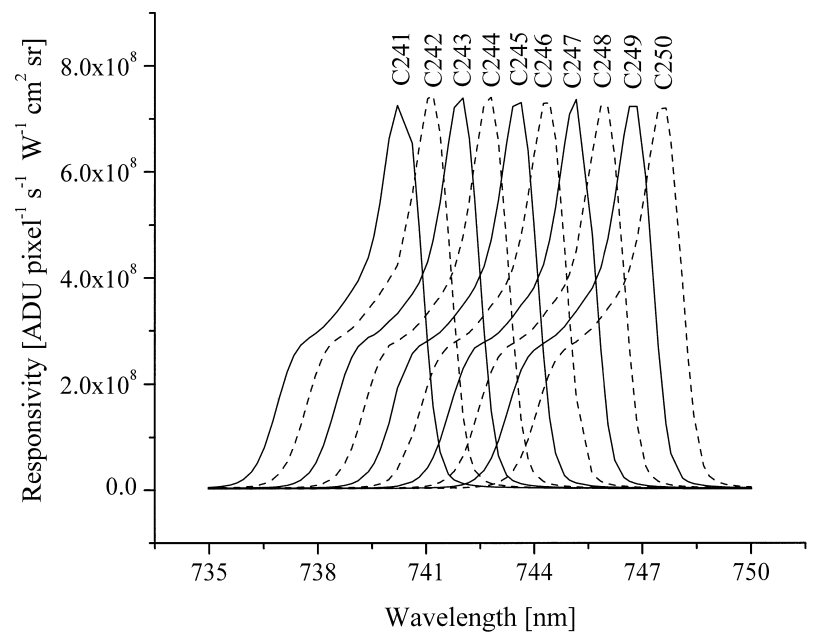

FIG. 8. The responsivity of columns $241-250$ as a function of wavelength acquired using the $0.2-\mathrm{nm}$ wavelength interval on SIRCUS. The solid and dashed lines are to assist the reader; they are not a fit to the data.

slit height. The effect of this curvature on the column radiance responsivity function was investigated by averaging over different number of rows around the center of the image of the slit (taken to be at row 325; see Fig. 7a). The behavior of $R_{245}\left(\lambda_{245}, \lambda^{\prime}\right)$ for illumination wavelengths close to $\lambda_{245}$ was determined by making measurements every $0.2 \mathrm{~nm}$ over a limited wavelength range-from 735 to $750 \mathrm{~nm}$. At each $\lambda^{\prime}, R_{245}\left(\lambda_{245}, \lambda^{\prime}\right)$ was determined. Figure $7 \mathrm{~b}$ shows the result averaged over different numbers of rows. Averaging over 50, 100, or 200 rows gives the same result. However, averaging over the full slit image (380 rows) gives a demonstrably different result, with decreased peak responsivity and a broadening to longer wavelengths. This is because of the image curvature. For the final results presented here, we averaged the image of the slit over the central 100 rows.

Figure 8 shows the result of the high-resolution scans from 735 to $750 \mathrm{~nm}$ for five sequential columns (column 241 to column 250). These correspond to the in-band values of $R_{i}\left(\lambda_{i}, \lambda^{\prime}\right)$ for these columns. The separation of the peaks is $0.8 \mathrm{~nm}$ and the full width at half maximum (FWHM) is $2.0 \mathrm{~nm}$. The shoulder on the short wavelength side is due to the asymmetry of the image of the slit.

For the full spectral calibration, images were acquired every $2-5 \mathrm{~nm}$ as the excitation wavelength was varied between 565 and $932 \mathrm{~nm}$. The wavelength step was too coarse to accurately determine each column's responsivity: $R_{i}\left(\lambda_{i}, \lambda^{\prime}\right)$. Therefore, we developed an algorithm to determine the peak wavelength $\left(\lambda_{i}\right)$ and maximum responsivity $\left[R_{i}\left(\lambda_{i}, \lambda_{i}\right)\right]$ of the column with the greatest response to excitation at a wavelength $\lambda^{\prime}$. The algorithm involved a comparison of the response of the column with the maximum response with the response of the two adjacent columns. From this comparison, the dif- 


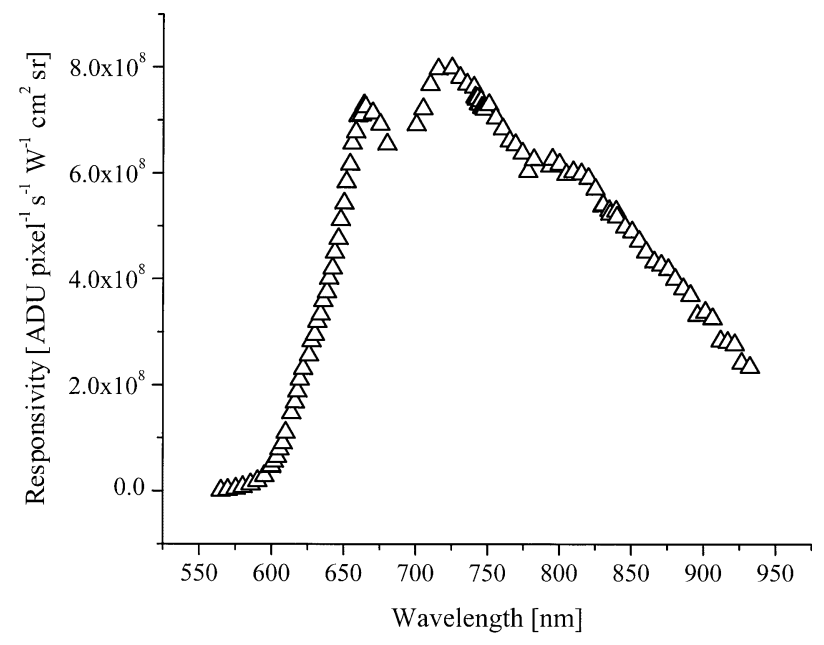

FIG. 9. The radiance responsivity of the red spectrograph determined using SIRCUS.

ference between the excitation wavelength and the column's peak response, $\lambda_{i}-\lambda^{\prime}$, could be determined. The maximum responsivity of column $i, R_{i}\left(\lambda_{i}, \lambda_{i}\right)$, was then determined by assuming the in-band shape is independent of $i$. To verify that the relative shape of the in-band component of $R_{i}\left(\lambda_{i}, \lambda^{\prime}\right)$ was invariant with respect to column (or wavelength), measurements on SIRCUS were also made over the spectral region from 640 to $652 \mathrm{~nm}$ with $0.2-\mathrm{nm}$ wavelength increments. The relative shape did not change.

The results for $R_{i}\left(\lambda_{i}, \lambda_{i}\right)$ are shown in Fig. 9. Under optimal conditions, the relative combined standard uncertainties for SIRCUS calibrations of single-detector radiometers are approximately $0.2 \%$ (Brown et al. 2000). This uncertainty is small because the radiance of the laser-illuminated sphere was determined accurately and because the single-detector radiometers are extremely stable. MOS measurements were made during multiple installations on SIRCUS. The repeatability near $775 \mathrm{~nm}$ was approximately 5\%, and increased to approximately $9 \%$ at $645 \mathrm{~nm}$. However, the bench unit was partially disassembled between measurements. Consequently, the large differences could be attributed to a constant change in the system throughput. Alternatively, they could be associated with the repeatability of the alignment of the MOS bench unit, in particular the rotating mirror that selects between the radiance and irradiance optics.

There is an additional $1 \%$ uncertainty from the algorithm used to determine the peak wavelength and maximum responsivity $R_{i}\left(\lambda_{i}, \lambda_{i}\right)$. Other uncertainty components for the MOS bench unit include the measurement noise, the degree of linearity, and the uncertainty associated with the correction with exposure time. These uncertainty components are typically small, on the order of $0.1 \%-0.3 \%$. We estimate that the SIRCUSderived values for $R_{i}\left(\lambda_{i}, \lambda_{i}\right)$ reported here have a relative expanded uncertainty of $5 \%(k=2)$.

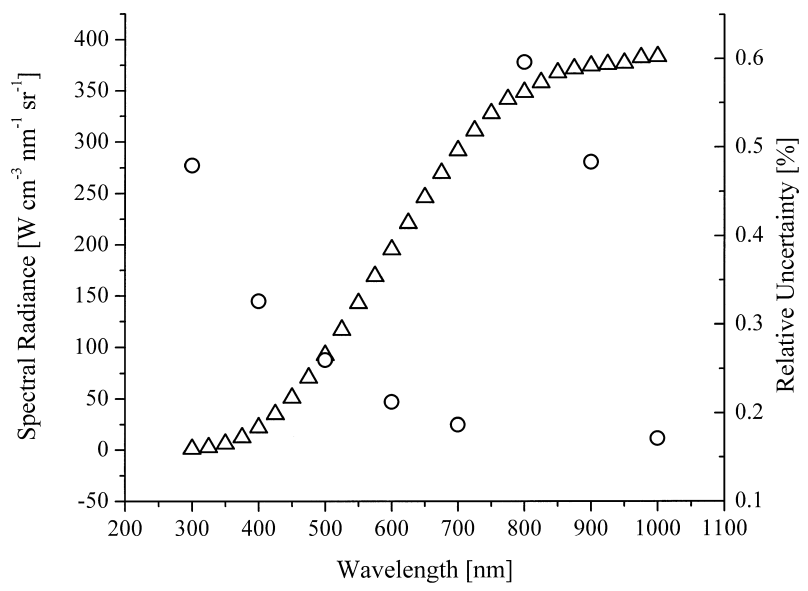

FIG. 10. The spectral radiance of the NPR (triangles) and the relative standard uncertainty (circles).

\section{Calibration using a broadband source}

The radiance responsivity of the MOS bench unit was determined in a manner similar to that employed by the MOBY team by using the NPR (Brown and Johnson 2002). The source is calibrated periodically for spectral radiance over the spectral range from 400 to $2400 \mathrm{~nm}$ at the primary U.S. facility for irradiance and radiance calibrations, the NIST Facility for Automated Spectroradiometric Calibrations (FASCAL; Walker et al. 1987). Consequently, the NPR is deployed to field experiments with a known spectral radiance along with established uncertainties. The NPR is equipped with two monitor photodiodes to verify that the sphere radiance remains stable during use.

The NPR ISS comes equipped with four 30-W lamps. For the calibration of MOS, a single lamp level was used. In Fig. 10, we show the NPR single-lamp level spectral radiance determined by FASCAL. Relative expanded uncertainties $(k=2)$ in the spectral radiance are less than $1 \%$ from 400 to $900 \mathrm{~nm}$. The MOS bench unit was positioned in front of the NPR and aligned to image the central region of the exit port. After a 30-min warmup of the NPR, images were acquired with a 0.5-s exposure time. Several images were acquired and averaged to determine the responsivity of the RSG. Monitor detector signals were recorded and used to verify the stability of the sphere radiance. Figure 11 shows a typical MOS RSG image of the NPR ISS. As with images recorded with monochromatic excitation sources (Fig. 3a), the image is slightly off center along the slit direction, with a maximum count rate along a particular column between rows 200 and 400. The count rate is low from columns 1 to 100 (for wavelengths from 550 to $625 \mathrm{~nm}$ ) and has a maximum value around column $225(725 \mathrm{~nm})$.

In the absence of detailed information on the shapes of $R_{i}\left(\lambda_{i}, \lambda\right)$ functions for all wavelengths, which is the case for the calibration of a MOBY or MOS systems by the MOBY team, the measurement equation [Eq. (1)] is approximated by 


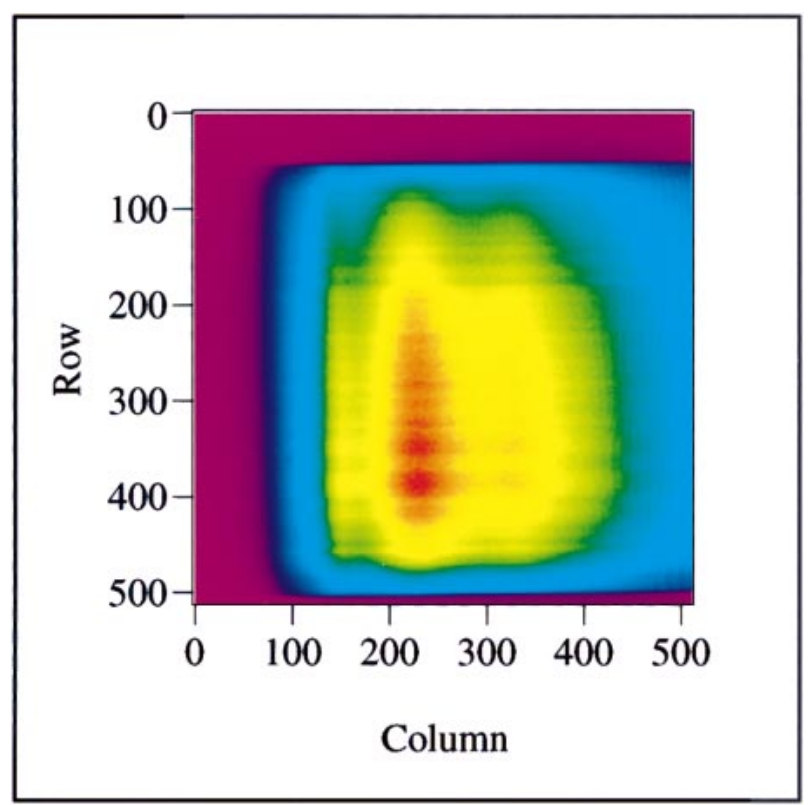

FIG. 11. A raw image (total counts) acquired by the RSG in $0.5 \mathrm{~s}$ using the NPR. A rainbow color table is used to represent the data, from blue (low) to red (high).

$$
F_{i}=\frac{S\left(\lambda_{i}\right)}{L_{\lambda}\left(\lambda_{i}\right) \Delta \lambda}
$$

where $F_{i}$ is the calibration factor for column $i$, and $L_{\lambda}\left(\lambda_{i}\right)$ is the spectral radiance (in $\mathrm{W} \mathrm{cm}-2 \mathrm{sr}^{-1} \mathrm{~nm}^{-1}$ ) of the broadband integrating sphere source, and $\Delta \lambda$ is the bandwidth of the RSG. The average count rate $S_{i}\left(\lambda_{i}\right)$ was found by averaging over rows $275-374$. The bandwidth, which is assumed to be independent of the column value $i$, is given by

$$
\Delta \lambda=\frac{\int R\left(\lambda_{246}, \lambda\right) d \lambda}{R\left(\lambda_{246}, \lambda_{246}\right)},
$$

which is just the area under the normalized, in-band responsivity function. For column $246, \Delta \lambda=2.53 \mathrm{~nm}$. The limits of the integration were determined by the span of the SIRCUS measurements at the 0.2-nm spacing.

Under optimal conditions, the relative combined standard uncertainties for broadband ISS-based calibrations of single-detector radiometers are $1 \%$ to $2 \%$ (Johnson et al. 2000). This uncertainty is larger than calibrations on SIRCUS because of the uncertainty components associated with the spectral radiance calibration of the ISS on FASCAL and effects such as temporal changes in the ISS output. The uniformity in spectral radiance at the exit aperture of the sphere is also important; for the NPR with four lamps illuminated, the spatial uniformity is within $\pm 0.3 \%$, with a $1 \%$ total variation over the entire exit aperture. The uniformity with only lamp 2 illuminated was not measured, but symmetry arguments say

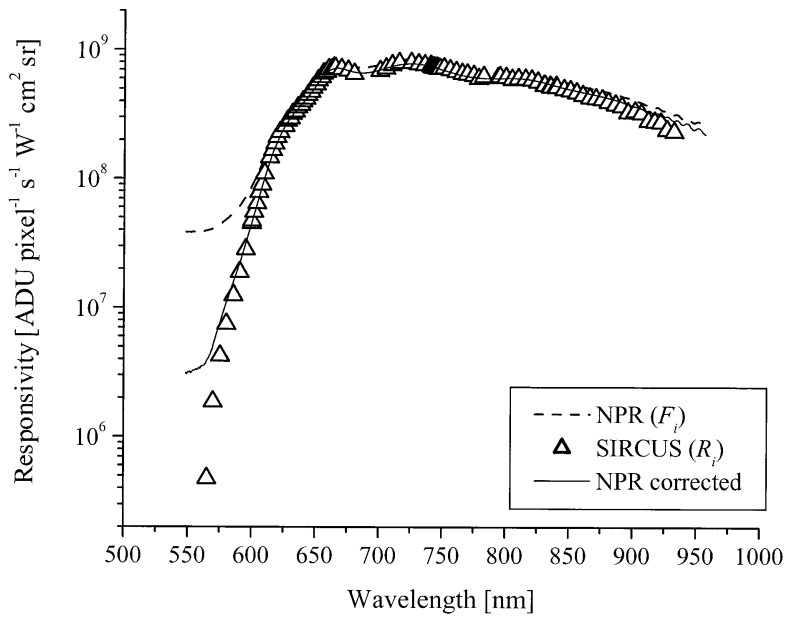

FIG. 12. The responsivity of the RSG with and without the correction for stray light applied to the NPR results.

the nonuniformity would be increased. Neglecting the uncertainty arising from stray light in the system, we estimate that the NPR-derived values for $F_{i}$ reported here have a relative expanded uncertainty of $3 \%(k=2)$.

\section{Discussion}

In Fig. 12, the responsivity $R_{i}\left(\lambda_{i}, \lambda_{i}\right)$ derived on SIRCUS is compared with the calibration factor $F_{i}$ determined by the lamp-illuminated sphere measurements. The spectral radiance responsivity determined by the lamp-illuminated sphere is approximately $10 \%$ higher than the responsivity determined on SIRCUS over the spectral range from 600 to $900 \mathrm{~nm}$. In the spectral region below $625 \mathrm{~nm}$, the two RSG responsivities diverge. The SIRCUS-derived responsivity tends to zero at $550 \mathrm{~nm}$ while the calibration factor determined using the NPR tends to a finite value.

We attribute the difference in the two responsivities to the out-of-band (or stray light) contribution to the total signal measured at each column $i$ in the broadband source calibration. As shown in Fig. 4b, there is a small but finite response at columns in the blue spectral region of the RSG to excitation light at $745 \mathrm{~nm}$. This finite response, when integrated over the spectral distribution of the excitation source, gives a contribution to the total signal that can dominate the total system response in regions of low responsivity (e.g., the 500-550-nm spectral range).

We have combined the results using the two approaches to develop a simple stray light correction algorithm for the NPR calibration (Brown et al. 2003). It is essentially a deconvolution approach (Shumaker 1979) that requires determination of the bandwidth for the in-band region and a description of the shape of the out-of-band response. Applying the correction algorithm to the NPR measurements results in a good agree- 
ment between the SIRCUS and the NPR calibration approaches, as shown in Fig. 12.

\section{Summary}

Results of a comprehensive radiometric study of one of two spectrographs in the MOS bench unit, the RSG, were presented. The spectral radiance responsivity of the spectrograph was measured using both a tunable narrowband laser-illuminated ISS and a broadband lamp-illuminated ISS. By comparing the two calibrations, we were able to separate the in-band and the outof-band (or stray light) contributions to the total system response. In particular, we showed that stray light introduces a significant error to measurements of broadband sources, such as the ISS calibration of the MOS bench unit responsivity, especially in regions where the response is small. Finally, we developed a simple algorithm based on a combination of the two calibration techniques to correct the broadband-source-based calibration of the MOS bench unit. Applying this stray light correction significantly reduced the discrepancy between the two calibration approaches.

Although demonstrated here for the RSG, similar effects are present in the BSG. In Brown et al. (2003), preliminary results for both spectrographs in the portable MOS that is deployed from ships (the MOS profiler) are described. The magnitude of the correction for the BSG is not the same as the RSG, but it is not negligible. These initial results indicate the stray light effect results in a bias in the derived $L_{u} \mathrm{~s}$ of between $3 \%$ and $5 \%$ in the blue and green spectral regions (Brown et al. 2003), with an estimated uncertainty in the correction algorithm of about $0.5 \%$. The stray light correction must be applied to all MOBY and MOS datasets. Work is in progress to characterize the remaining instruments in the MOBY program in order to determine the correction algorithm parameters; this will allow for reprocessing of all of the MOBY datasets, including those used to validate MODIS, SeaWiFS, and other ocean color satellites. The stray light correction algorithm applied to both the calibration and subsequent in-water measurements will reduce the uncertainty in measurements of upwelling radiance and downwelling irradiance, resulting in better estimates of global chlorophyll- $a$ and other ocean color data products.

Acknowledgments. The authors acknowledge support for this work from the National Oceanic and Atmospheric Administration and the U.S. Air Force Metrology.

\section{REFERENCES}

Barnes, R. A., and A. W. Holmes, 1993: Overview of the SeaWiFS ocean sensor. Proc. SPIE, 1939, 224-232.
R. E. Eplee Jr., W. D. Robinson, G. M. Schmidt, F. S. Patt, S. W. Bailey, M. Wang, and C. R. McClain, 2000: The calibration of SeaWiFS on orbit. Proc. SPIE, 4135, 281-293.

Brown, S. W., and B. C. Johnson, 2003: Development of a portable integrating sphere source for the Earth Observing System's calibration validation program. Int. J. Remote Sens., in press.

- G. P. Eppeldauer, and K. R. Lykke, 2000: NIST facility for Spectral Irradiance and Radiance Responsivity Calibrations with Uniform Source. Metrologia, 37, 579-582.

— B B. C. Johnson, M. E. Feinholz, M. A. Yarbrough, S. J. Flora, K. R. Lykke, and D. K. Clark, 2003: Stray light correction algorithm for spectrographs. Metrologia, in press.

Butler, J. J., and Coauthors, 1999: Radiometric measurement comparisons using transfer radiometers in support of the calibration of NASA's Earth Observing System (EOS) sensors. Proc. SPIE, 3870, 180-192.

Clark, D. K., H. R. Gordon, K. J. Voss, Y. Ge, W. Broenkow, and C. Trees, 1997: Validation of atmospheric correction over the oceans. J. Geophys. Res., 102, 17 209-17 217.

—- M. E. Feinholz, M. A. Yarbrough, B. C. Johnson, S. W. Brown, Y. S. Kim, and R. A. Barnes, 2002a: An overview of the radiometric calibration of MOBY. Proc. SPIE, 4483, 64-76.

- and Coauthors, 2002b: MOBY, A radiometric buoy for performance monitoring and vicarious calibration of satellite ocean color sensors: Measurement and data analysis protocols. Ocean optics protocols for satellite ocean color sensor validation, revision 3, Vol. 2. Tech. Memo. NASA/TM-2002-210004/Rev3Vol 2, NASA Goddard Space Flight Center, 138-170.

Deschamps, P. Y., F. M. Breon, M. Leroy, A. Podaire, A. Bricaud, J. C. Buriez, and G. Seze, 1994: The POLDER mission: Instrument characteristics and scientific objectives. IEEE Trans. Geosci. Remote Sens., 32, 598-615.

Elachi, C., 1987: Introduction to the Physics and Techniques of Remote Sensing. John Wiley and Sons, $413 \mathrm{pp}$.

Esaias, W. E., and Coauthors, 1998: An overview of MODIS capabilities for ocean science observations. IEEE Trans. Geosci. Remote Sens., 36, 1250-1265.

Gordon, H. R., 1998: In-orbit calibration strategy for ocean color sensors. Remote Sens. Environ., 63, 265-278.

- D. K. Clark, J. L. Mueller, and W. A. Hovis, 1980: Phytoplankton pigments derived from the Nimbus-7 CZCS: Initial comparisons with surface measurements. Science, 210, 63-66.

Hooker, S. B., C. R. McClain, and A. Holmes, 1993: Ocean color imaging: CZCS to SeaWiFS. Marine Tech. Soc. Journal, 27, 3 15.

Isaacman, A., B. Franz, and R. E. Eplee Jr., 1999: An investigation of time variability in water-leaving radiances retrieved from ocean color measurements. Proc. ALPS99 Conf., Meribel, France, Centre Nationale d'Etudes Spatiales, 18-22.

Johnson, B. C., S. W. Brown, and H. W. Yoon, 2000: Radiometric calibration history of visible and near-infrared portable radiometers. Metrologia, 37, 423-426.

Kostkowski, H. J., 1979: The relative spectral responsivity and slitscatter function of a spectroradiometer. Self study manual on optical radiation measurements, NBS Tech. Note 910-4, U.S. Dept. Commerce, National Bureau of Standards, 1-34.

Shumaker, J. B., 1979: Deconvolution. Self study manual on optical radiation measurements, NBS Tech. Note 910-4, U.S. Dept. Commerce, National Bureau of Standards, 35-90.

Walker, J. H., R. D. Saunders, and A. T. Hattenburg, 1987: Spectral Radiance Calibrations. NBS Special Publication 250-1, U.S. Government Printing Office, 26 pp. (Plus appendices.)

Wang, M., A. Isaacman, B. A. Franz, and C. R. McClain, 2002: Ocean-color optical property data derived from the Japanese Ocean Color and Temperature Scanner and the French Polarization and Directionality of the Earth's Reflectances: A comparison study. Appl. Optics, 41, 974-990. 